\title{
Multikinase-Inhibitor Screening in Drug-resistant Osteosarcoma Patient-derived Orthotopic Xenograft Mouse Models Identifies the Clinical Potential of Regorafenib
}

\author{
TAKASHI HIGUCHI ${ }^{1,2,3}$, KENTARO IGARASHI ${ }^{3}$, NORIO YAMAMOTO ${ }^{3}$, \\ KATSUHIRO HAYASHI ${ }^{3}$, HIROAKI KIMURA ${ }^{3}$, SHINJI MIWA ${ }^{3}$, \\ MICHAEL BOUVET ${ }^{2}$, HIROYUKI TSUCHIYA ${ }^{3}$ and ROBERT M. HOFFMAN ${ }^{1,2}$ \\ ${ }^{1}$ AntiCancer, Inc., San Diego, CA, U.S.A.; \\ ${ }^{2}$ Department of Surgery, University of California, San Diego, CA, U.S.A.; \\ ${ }^{3}$ Department of Orthopaedic Surgery, Graduate School of Medical Sciences, Kanazawa University, Kanazawa, Japan
}

\begin{abstract}
Background/Aim: Osteosarcoma is a recalcitrant heterogenous malignancy. The aim of the present study was to compare a series of multikinase inhibitors (MKIs) for efficacy on two drug-resistant osteosarcoma patient-derived orthotopic xenograft (PDOX) models in order to identify a clinical candidate. Materials and Methods: The two osteosarcoma PDOX models were tested for response to the following MKIs: pazopanib, sunitinib, sorafenib, crizotinib, and regorafenib, in comparison to first-line treatment with cisplatinum and an untreated control. Results: Regorafenib led to regression of osteosarcoma in both PDOXs. Total necrosis was observed pathologically in the regorafenib-treated tumors. Sorafenib arrested growth, without inducing regression, in one osteosarcoma model but not the other, and the other MKIs only slowed tumor growth. Conclusion: The present study demonstrated that regorafenib is much more effective than the other MKIs tested and has clinical potential against recalcitrant osteosarcoma.
\end{abstract}

Osteosarcoma is a highly-malignant primary bone tumor arising from heterogeneous transformed cells of mesenchymal origin within the skeleton. The rarity and heterogeneity of this disease has limited the discovery of improved treatment options $(1,2)$.

This article is freely accessible online.

Correspondence to: Robert M. Hoffman, AntiCancer, Inc., 7917 Ostrow St., San Diego, CA 92111, U.S.A. E-mail: all@anticancer.com and Hiroyuki Tsuchiya, Department of Orthopaedic Surgery, Graduate School of Medical Sciences, Kanazawa University, 13-1 Takaramachi, Kanazawa, Ishikawa 9208641, Japan. E-mail: tsuchi@med.kanazawa-u.ac.jp

Key Words: Osteosarcoma, multikinase-inhibitors, regorafenib, patient-derived orthotopic xenograft, screening.
In recent years, multikinase inhibitors (MKIs) such as sunitinib, sorafenib, pazopanib, crizotinib and regorafenib have shown promising results, initially in renal-cell cancer (RCC) (3), hepatocellular carcinoma (HCC) (4), and thyroid cancer (5). MKIs simultaneously target several kinases (6). Preclinical results have indicated the potential efficacy of MKIs against osteosarcoma; however, there is presently no approved MKI for first-line treatment of osteosarcoma $(7,8)$.

We developed patient-derived orthotopic xenograft (PDOX) models of all major types of malignancies, including osteosarcoma, with the technique of surgical orthotopic implantation (SOI), allowing effective and ineffective drugs to be distinguished (9-13). The PDOX models recapitulate human-tumor behavior better than other in vivo models due to their intact histology and correct organ-tumor microenvironment of the orthotopicallyimplanted tumor (14-16). Recently, we demonstrated that sorafenib, which targets intracellular kinase activity of vascular endothelial growth factor receptors (VEGFRs), inhibited chemotherapy-resistant osteosarcoma in PDOX models, indicating its potential for the treatment of osteosarcoma $(17,18)$.

The present study screened a panel of MKIs in two independent osteosarcoma PDOX models, in order to identity the best clinical candidate.

\section{Materials and Methods}

Mice. Athymic nude mice, 4-6 weeks old, were used. Mouse housing, experimental procedures and data collection were conducted as described in our previous publications $(9-13,15,17$, 18). All surgical procedures were performed under appropriate anesthesia and analgesics to minimize any suffering of the animals in accordance with the principles and procedures summarized in the National Institutes of Health Guide for the Care and Use of 
A

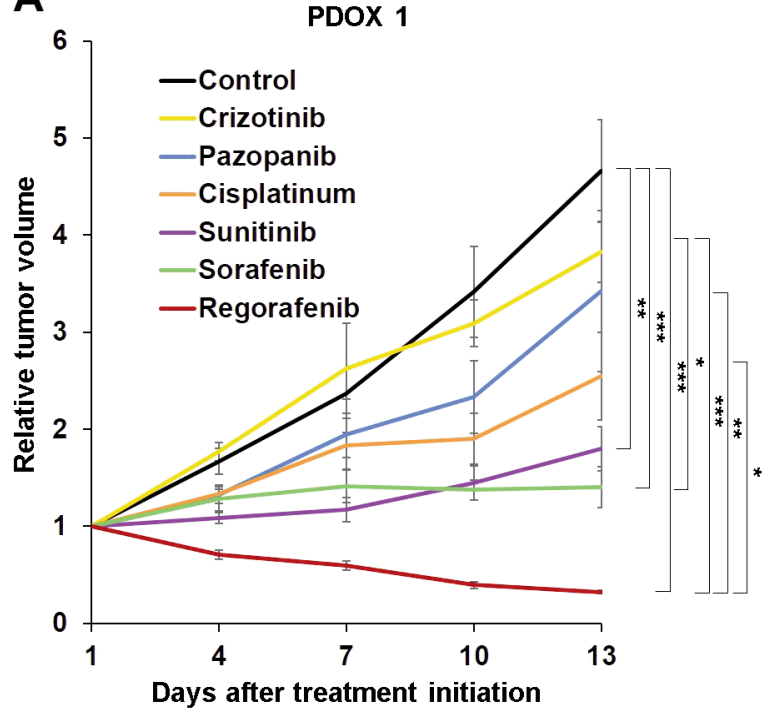

B

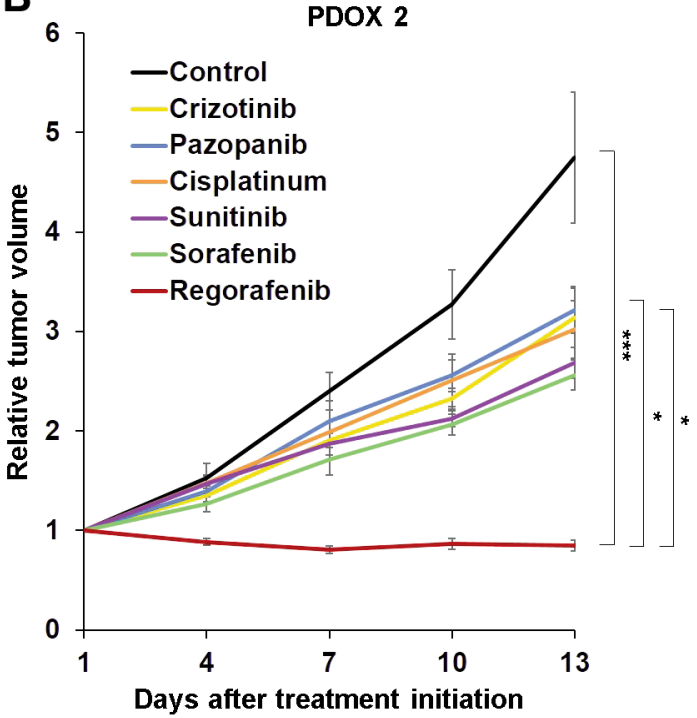

Figure 1. Efficacy of multikinase inhibitors (MKIs) on osteosarcoma patient-derived orthotopic xenograft (PDOX) mouse models. Line graphs show tumor volume at time points after treatment initiation relative to tumor volume at the beginning of treatment. A: Osteosarcoma PDOX established from a tumor of a patient with conventional pelvic osteosarcoma (PDOX 1). B: Osteosarcoma PDOX established from a bone metastasis from a patient who had undergone resection of primary osteosarcoma of the left femur (PDOX 2). Data are mean \pm standard error of mean (SEM). Significantly different at: $* p<0.05, * * p<0.01$, and $* * * p<0.001$.

Animals under Assurance Number A3873-1. An AntiCancer, Institutional Animal Care and Use Committee protocol specifically approved this study.

Patient-derived tumors for establishment of PDOX models. PDOX 1: A biopsy sample was obtained from a 14-year-old patient who presented with conventional osteosarcoma of the pelvis. Neither chemotherapy nor radiotherapy was performed before biopsy (9). PDOX 2: A surgical sample of the metastatic lesion of the left tibia was obtained from a 23-year-old patient who had been diagnosed with conventional osteosarcoma of the femur and subsequently presented with lung metastasis after failure of cisplatinum-based chemotherapy. Written informed consent was provided from the patients and their parents under an Institutional Review Boardapproved protocol of University of California, Los Angeles (IRB \#10-001857).

These fresh tumor samples were previously transported to the laboratory on ice and established in nude mice using small tumor fragments transplanted subcutaneously $(10,11,17-19)$.

Osteosarcoma PDOX models. The subcutaneously-implanted tumors, grown to more than $10 \mathrm{~mm}$ in diameter, were resected and small fragments $(2-3 \mathrm{~mm})$ were made from the resected tumors. SOI of the tumor fragments into nude mice was performed as previously described: A 10-15 mm skin incision was made on the lateral side of the distal thigh. The vastus lateralis muscle and biceps femoris muscle were split, and the lateral patello-femoral ligament was cut to reach the distal femur. The lateral condyle of the femur was resected with scissors to create a space. A single tumor fragment was inserted into this space and the muscle as well as the skin were sutured with 6-0 nylon $(9,17-19)$.
Study drugs. The following drugs were used in the present study: Cisplatinum (Bristol Myers Squibb, New York, NY, USA) a standard first-line treatment for osteosarcoma; pazopanib (GlaxoSmithKline Plc., London, UK), an MKI approved for softtissue sarcoma and RCC (20); sunitinib (Pfizer Inc., New York, NY, USA), an MKI approved for gastrointestinal stromal tumors (GIST) and RCC (21); sorafenib (Bayer AG, Leverkusen, Germany), an MKI approved for RCC, HCC, and thyroid carcinoma (22); crizotinib (Pfizer Inc.), an MKI approved for non-small-cell lung cancer and anaplastic large-cell lymphoma (23); and regorafenib (Bayer AG), an MKI approved for metastatic colorectal cancer, GIST, and metastatic HCC (24).

Treatment study-design. The established osteosarcoma PDOX models were divided into seven treatment groups of six mice each: Group 1: Control, treated orally daily with phosphatebuffered saline at $100 \mu \mathrm{l} / \mathrm{mouse}$; group 2: $6 \mathrm{mg} / \mathrm{kg}$ cisplatinum injected intraperitoneally once a week; group 3: $50 \mathrm{mg} / \mathrm{kg}$ pazopanib orally daily; group 4: $40 \mathrm{mg} / \mathrm{kg}$, sunitinib orally daily; group 5: $30 \mathrm{mg} / \mathrm{kg}$ sorafenib orally daily; group $6: 25 \mathrm{mg} / \mathrm{kg}$ crizotinib orally daily; group 7: $30 \mathrm{mg} / \mathrm{kg}$ regorafenib orally daily. Treatment started 3 weeks after SOI and lasted 2 weeks. Tumor diameter and mouse body-weight were monitored with a digital caliper and a digital scale, respectively, twice a week. The formula [tumor volume $\left(\mathrm{mm}^{3}\right)=$ long diameter $(\mathrm{mm}) \times$ short diameter $(\mathrm{mm}) \times$ short diameter $(\mathrm{mm}) \times 0.5]$ was used for tumorvolume calculation.

Tumor histology. Resected tumors were embedded in paraffin after adequate fixation in formalin followed by sectioning and staining. After deparaffinization and rehydration of the tissue sections in 

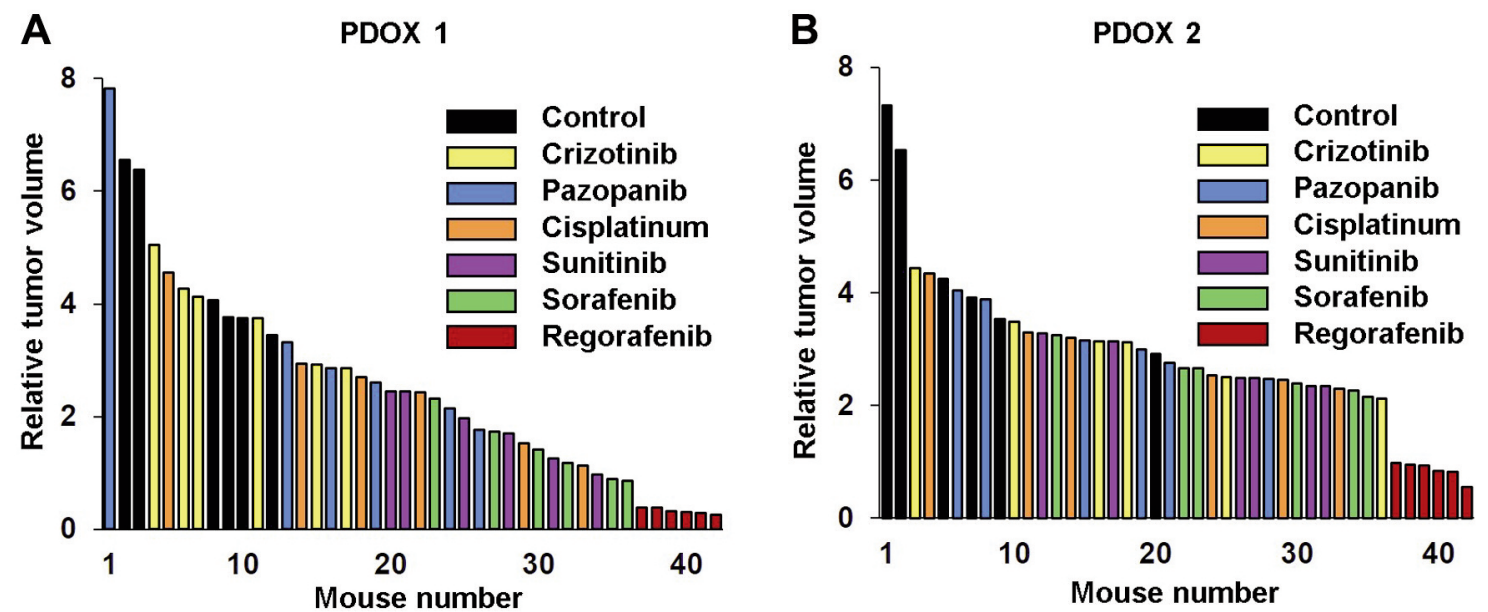

Figure 2. Waterfall plot of tumor volume at the end of treatment relative to tumor volume at the beginning of treatment for each osteosarcoma patient-derived orthotopic xenograft (PDOX) mouse model. A: PDOX 1. B: PDOX 2. Data are mean \pm standard error of mean (SEM). Significantly different at: $* p<0.05, * * p<0.01$, and $* * * p<0.001$.

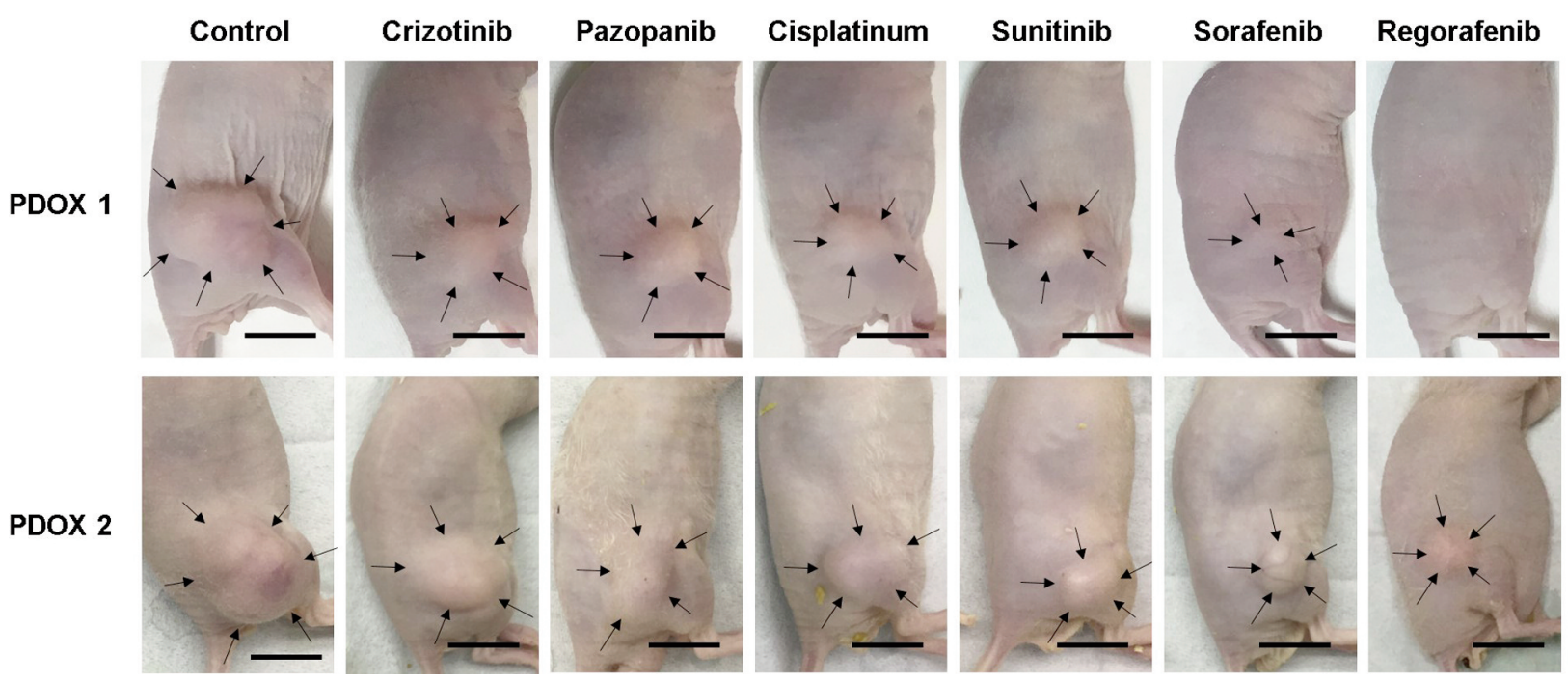

Figure 3. Representative images of osteosarcoma patient-derived orthotopic xenograft mouse models PDOX 1 and PDOX 2 at the completion of treatment. Arrows indicate the tumor margins. Scale bars: $10 \mathrm{~mm}$.

xylene and ethanol, the sections were stained with hematoxylin and eosin. Histological responses to the drugs, including cancer necrosis and cancer-cell density, were evaluated.

Statistical analysis. One-way analysis of variance or a paired $t$-test using EZR statistical software (Jichi Medical University Saitama Medical Center, Saitama, Japan) were used for statistical analyses. Data are shown as the mean \pm standard error of the mean. All $p$ values were two-sided and values of $p \leq 0.05$ were considered significant.

\section{Results}

Efficacy of MKIs in the osteosarcoma PDOX models. Only regorafenib led to tumor regression in both osteosarcoma PDOX models and apparent tumor eradication in PDOX 1 (Figures 1-3). Tumor size was significantly lower in mice treated with regorafenib compared to the control, and crizotinib- and pazopanib-treated mice in both osteosarcoma PDOX models and compared to cisplatinum only in PDOX 

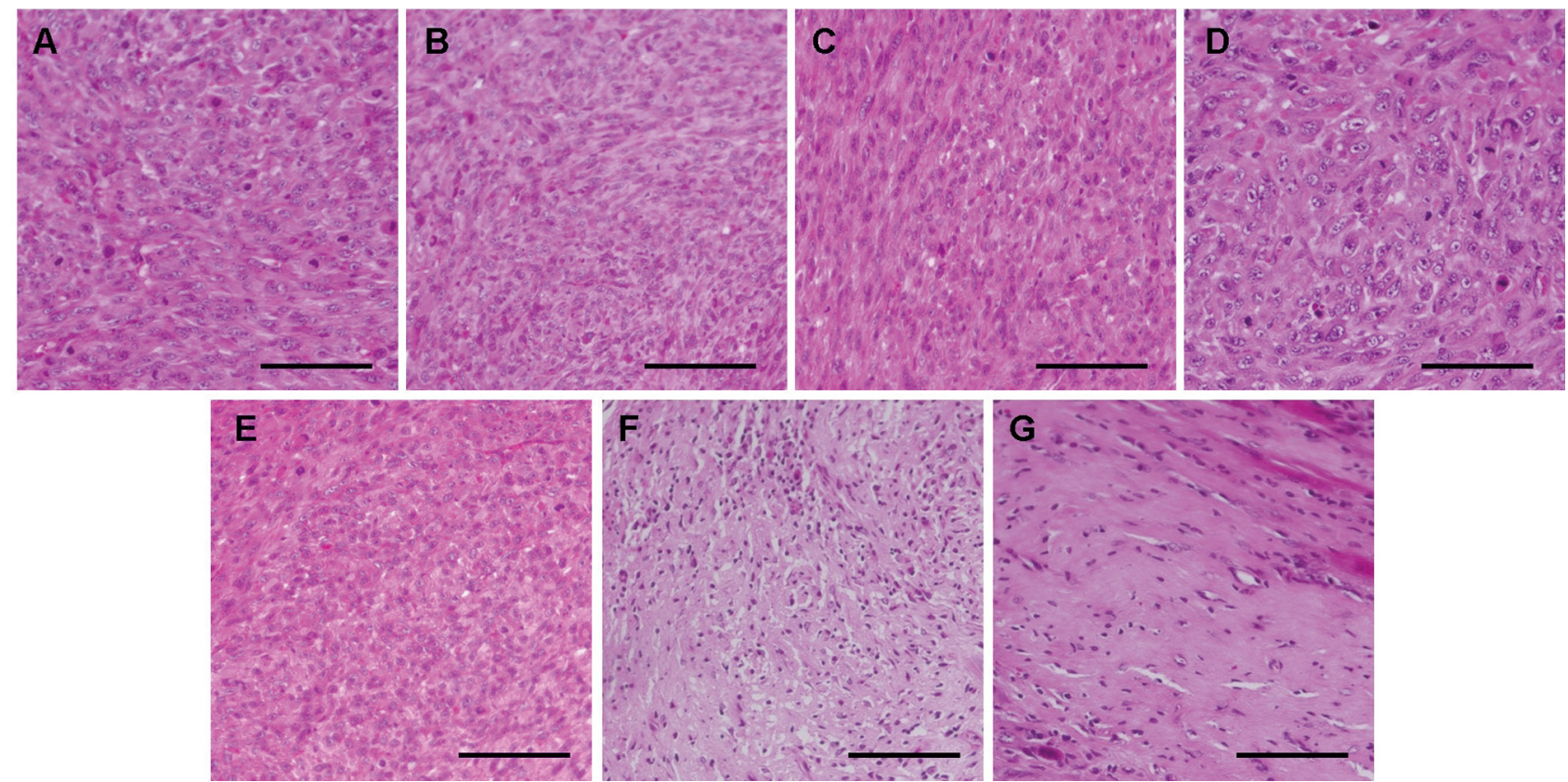

Figure 4. Hematoxylin and eosin-stained sections of tumors resected from osteosarcoma patient-derived orthotopic xenograft (PDOX 1) mouse models under different treatments. A: Control. B: Crizotinib C: Pazopanib. D: Cisplatinum. E: Sunitinib. F: Sorafenib. G: Regorafenib. Scale bars: 100 um.
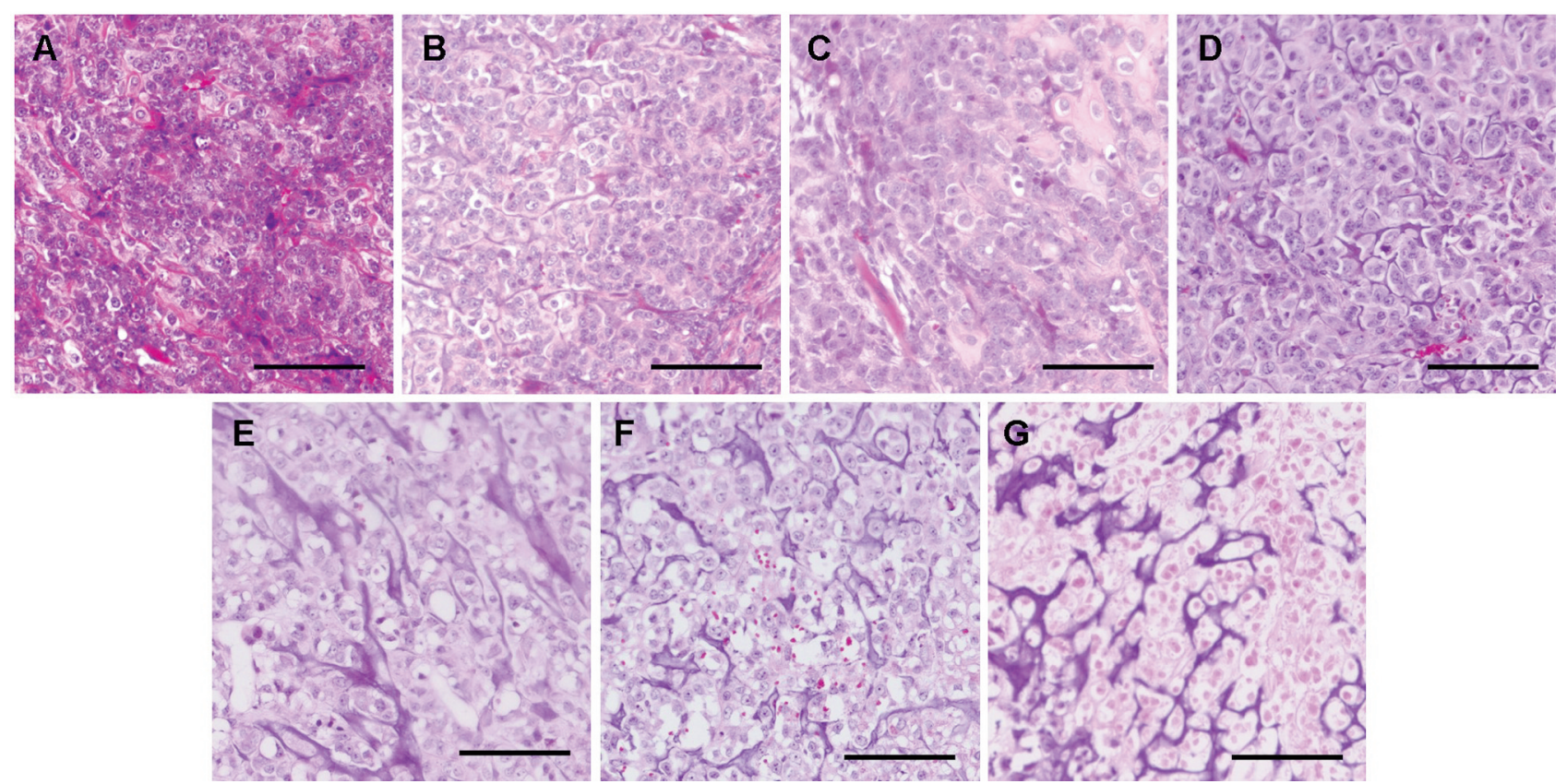

Figure 5. Hematoxylin and eosin-stained sections of tumors resected from osteosarcoma patient-derived orthotopic xenograft mouse models (PDOX 2) under different treatments. A: Control. B: Crizotinib. C: Pazopanib. D: Cisplatinum. E: Sunitinib. F: Sorafenib. G: Regorafenib. Scale bars: 100 um. 


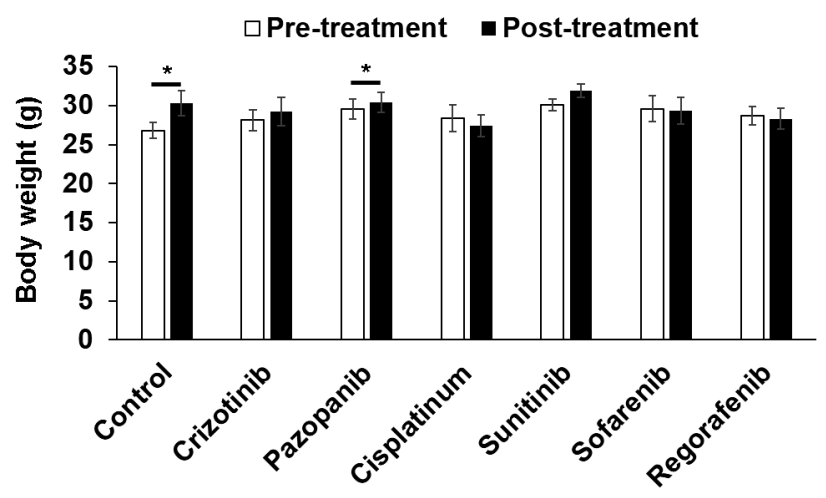

Figure 6. Body weight of osteosarcoma patient-derived orthotopic xenograft mouse models (PDOX) before and after different treatments. A: Control. B: Crizotinib. C: Pazopanib. D: Cisplatinum. E: Sunitinib. F: Sorafenib. G: Regorafenib. Scale bars: $100 \mu \mathrm{m}$. Data are mean \pm standard error of mean (SEM). Significantly different at: $* p<0.05$.

1. Sorafenib and sunitinib significantly inhibited tumor growth in PDOX 1 compared to the control, but not in PDOX 2 (Figures 1-3).

Histology of the osteosarcoma PDOX models. In both PDOX models, control tumors comprised dense, viable cancer cells with many mitoses and osteoid formation. Tumors treated with crizotinib, pazopanib, or cisplatinum in both PDOX models, and those in PDOX 1 treated with sunitinib, exhibited a lower density of viable cancer cells than in control mice. Tumors treated with sunitinib in PDOX 2 and sorafenib in both models comprised a small number of viable cancer cells and calcification or fibrosis of stromal tissue. Total necrosis, with many calcifications and fibrosis, was found in tumors treated with regorafenib in both PDOX models (Figure 4 and Figure 5).

Effects of MKIs on mouse survival and body-weight in the osteosarcoma PDOX models. No mouse died in any group. There was a significant increase in mouse body-weight in control $(p=0.04)$ and pazopanib-treated $(p=0.03)$ mice. No significant body-weight change was found in the other groups (Figure 6).

\section{Discussion}

The present study demonstrated that out of the five MKIs screened, only regorafenib induced tumor regression and total necrosis in two independent, drug-resistant PDOX models of osteosarcoma which were resistant to first-line cisplatinum.

We developed the PDOX model for all major cancer types, including osteosarcoma, and have demonstrated the efficacy of many approved and experimental chemotherapy drugs indicated for other malignancies, for precision medicine, which is especially important for rare types of cancer $(9-13,15,17,18)$. We recently reported that combination treatment including sorafenib, an MKI, is effective against osteosarcoma in PDOX models, suggesting the potential usefulness of MKIs for treating osteosarcoma $(17,18)$. MKIs prevent progression of many types of cancer by inhibiting multiple hyper-activated-receptor kinases, such as fibroblast-growth-factor receptor, VEGFR, and stem-cell factor (c-KIT) $(5,6,8,25)$. Abnormality of these receptor kinases has also been reported in sarcoma. Pazopanib has been used for advanced soft-tissue sarcoma, with limited efficacy $(26,27)$. For osteosarcoma treatment in the clinic, no MKI is currently approved, despite many receptor-kinase abnormalities associated with osteosarcoma, probably due to the rarity and heterogeneity of the disease which leads to difficulty in accumulating clinical and pre-clinical evidence. In the present study, we found regorafenib was the most effective MKI among multiple MKIs tested on osteosarcoma PDOX models. Regorafenib, an oral small-molecule MKI, targets angiogenic (VEGFRs, angiopoietin-1 and -2), stromal (platelet-derived growth factor receptor $\beta$, fibroblast growth factor receptor), and oncogenic- receptor tyrosine kinases (c-KIT, rapidly-accelerated fibrosarcoma [RAF], and rearranged during transfection $[\mathrm{RET}])(26,28)$. Regorafenib was initially approved for the treatment of metastatic colorectal cancer, and subsequently for HCC and GIST, but it has not been approved for sarcoma $(26,28)$. We previously reported that regorafenib led to regression of a chest-wall-metastatic Ewing's sarcoma in a PDOX model, suggesting it may be effective for other sarcoma types (29). Tumor regression and total necrosis caused by regorafenib were observed in two different osteosarcoma PDOX models in the present study, suggesting the clinical potential of regorafenib for recalcitrant osteosarcoma. Common mechanisms of drug resistance and sensitivity observed among the osteosarcoma PDOX models will be determined in future studies.

\section{Conflicts of Interest}

The Authors declare no competing interests.

\section{Authors' Contributions}

Conception and design: TH and RMH. Acquisition, analysis, and interpretation of data: TH, KI, NY, KH, HK, SM, and MB. Writing, review, and revision of the article: TH, HT, and RMH.

\section{Acknowledgements}

This paper is dedicated to the memory of A.R. Moossa, MD, Sun Lee, MD, Professor Li Jiaxi and Masaki Kitajima, MD. 


\section{References}

1 Belayneh R, Fourman MS, Bhogal S and Weiss KR: Update on Osteosarcoma. Curr Oncol Rep 23(6): 71, 2021. PMID: 33880674. DOI: 10.1007/s11912-021-01053-7

2 Gazouli I, Kyriazoglou A, Kotsantis I, Anastasiou M, Pantazopoulos A, Prevezanou M, Chatzidakis I, Kavourakis G, Economopoulou P, Kontogeorgakos V, Papagelopoulos P and Psyrri A: Systematic review of recurrent osteosarcoma systemic therapy. Cancers (Basel) 13(8): 1757, 2021. PMID: 33917001. DOI: $10.3390 /$ cancers 13081757

3 Leung HW and Chan AL: Multikinase inhibitors in metastatic renal cell carcinoma: indirect comparison meta-analysis. Clin Ther 33(6): 708-716, 2011. PMID: 21704235. DOI: 10.1016/ j.clinthera.2011.05.003

4 Chen S, Cao Q, Wen W and Wang H: Targeted therapy for hepatocellular carcinoma: Challenges and opportunities. Cancer Lett 460: 1-9, 2019. PMID: 31207320. DOI: 10.1016/j.canlet. 2019.114428

5 Gild ML, Tsang VHM, Clifton-Bligh RJ and Robinson BG: Multikinase inhibitors in thyroid cancer: timing of targeted therapy. Nat Rev Endocrinol 17(4): 225-234, 2021. PMID: 33603220. DOI: 10.1038/s41574-020-00465-y

6 Ancker OV, Krüger M, Wehland M, Infanger M and Grimm D: Multikinase inhibitor treatment in thyroid cancer. Int J Mol Sci 21(1): 10, 2019. PMID: 31861373. DOI: 10.3390/ijms21010010

7 Harrison DJ, Gill JD, Roth ME, Zhang W, Teicher B, Erickson S, Gatto G, Kurmasheva RT, Houghton PJ, Smith MA, Kolb EA and Gorlick R: Initial in vivo testing of a multitarget kinase inhibitor, regorafenib, by the Pediatric Preclinical Testing Consortium. Pediatr Blood Cancer 67(6): e28222, 2020. PMID: 32207565. DOI: $10.1002 /$ pbc.28222

8 Tian Z, Niu X and Yao W: Receptor tyrosine kinases in osteosarcoma treatment: Which is the key target? Front Oncol 10: 1642, 2020. PMID: 32984034. DOI: 10.3389/fonc.2020.01642

9 Higuchi T, Yamamoto J, Sugisawa N, Tashiro Y, Nishino H, Yamamoto N, Hayashi K, Kimura H, Miwa S, Igarashi K, Bouvet M, Singh SR, Tsuchiya H and Hoffman RM: PPAR $\gamma$ agonist pioglitazone in combination with cisplatinum arrests a chemotherapy-resistant osteosarcoma PDOX model. Cancer Genomics Proteomics 17(1): 35-40, 2020. PMID: 31882549. DOI: $10.21873 / \operatorname{cgp} .20165$

10 Igarashi K, Kawaguchi K, Yamamoto N, Hayashi K, Kimura H, Miwa S, Higuchi T, Taniguchi Y, Yonezawa H, Araki Y, Morinaga S, Misra S, Nelson SD, Dry SM, Li Y, Odani A, Singh SR, Tsuchiya $\mathrm{H}$ and Hoffman RM: A novel anionic-phosphateplatinum complex effectively targets a cisplatinum-resistant osteosarcoma in a patient-derived orthotopic xenograft mouse model. Cancer Genomics Proteomics 17(3): 217-223, 2020. PMID: 32345663. DOI: $10.21873 / \mathrm{cgp} .20182$

11 Oshiro H, Tome Y, Miyake K, Higuchi T, Sugisawa N, Kanaya F, Nishida K and Hoffman RM: An mTOR and VEGFR inhibitor combination arrests a doxorubicin resistant lung metastatic osteosarcoma in a PDOX mouse model. Sci Rep 11(1): 8583, 2021. PMID: 33883561. DOI: 10.1038/s41598-021-87553-9

12 Sugisawa N, Higuchi T, Han Q, Hozumi C, Yamamoto J, Tashiro Y, Nishino H, Kawaguchi K, Bouvet M, Murata T, Unno M and Hoffman RM: Oral recombinant methioninase combined with paclitaxel arrests recalcitrant ovarian clear cell carcinoma growth in a patient-derived orthotopic xenograft (PDOX) nude-mouse model. Cancer Chemother Pharmacol 88(1): 61-67, 2021. PMID: 33768300. DOI: $10.1007 / \mathrm{s} 00280-021-04261-x$

13 Zhang Z, Hu K, Miyake K, Kiyuna T, Oshiro H, Wangsiricharoen S, Kawaguchi K, Higuchi T, Razmjooei S, Miyake M, Chawla SP, Singh SR and Hoffman RM: A novel patient-derived orthotopic xenograft (PDOX) mouse model of highly-aggressive liver metastasis for identification of candidate effective drug-combinations. Sci Rep 10(1): 20105, 2020. PMID: 33208807. DOI: 10.1038/s41598-020-76708-9

14 DeRose YS, Wang G, Lin YC, Bernard PS, Buys SS, Ebbert MT, Factor R, Matsen C, Milash BA, Nelson E, Neumayer L, Randall RL, Stijleman IJ, Welm BE and Welm AL: Tumor grafts derived from women with breast cancer authentically reflect tumor pathology, growth, metastasis and disease outcomes. Nat Med 17(11): 1514-1520, 2011. PMID: 22019887. DOI: 10.1038/nm.2454

15 Kiguchi K, Kubota T, Aoki D, Udagawa Y, Yamanouchi S, Saga M, Amemiya A, Sun FX, Nozawa S, Moossa AR and Hoffman RM: A patient-like orthotopic implantation nude mouse model of highly metastatic human ovarian cancer. Clin Exp Metastasis 16(8): 751756, 1998. PMID: 10211988. DOI: 10.1023/a:1006537013317

16 Zheng D: Orthotopic tumours, a hot topic for xenograft models? EBioMedicine 41: 11-12, 2019. PMID: 30833189. DOI: 10.1016/j.ebiom.2019.02.052

17 Higuchi T, Sugisawa N, Miyake K, Oshiro H, Yamamoto N, Hayashi K, Kimura H, Miwa S, Igarashi K, Chawla SP, Bouvet M, Singh SR, Tsuchiya H and Hoffman RM: Sorafenib and palbociclib combination regresses a cisplatinum-resistant osteosarcoma in a PDOX mouse model. Anticancer Res 39(8): 4079-4084, 2019. PMID: 31366491. DOI: 10.21873/anticanres.13565

18 Higuchi T, Sugisawa N, Miyake K, Oshiro H, Yamamoto N, Hayashi K, Kimura H, Miwa S, Igarashi K, Kline Z, Belt P, Chawla SP, Bouvet M, Singh SR, Tsuchiya H and Hoffman RM: Combination treatment with sorafenib and everolimus regresses a doxorubicin-resistant osteosarcoma in a PDOX mouse model. Anticancer Res 39(9): 4781-4786, 2019. PMID: 31519579. DOI: 10.21873/anticanres.13662

19 Higuchi T, Oshiro H, Miyake K, Sugisawa N, Han Q, Tan Y, Park J, Zhang Z, Razmjooei S, Yamamoto N, Hayashi K, Kimura H, Miwa S, Igarashi K, Bouvet M, Chawla SP, Singh SR, Tsuchiya $\mathrm{H}$ and Hoffman RM: Oral recombinant methioninase, combined with oral caffeine and injected cisplatinum, overcome cisplatinumresistance and regresses patient-derived orthotopic xenograft model of osteosarcoma. Anticancer Res 39(9): 4653-4657, 2019. PMID: 31519563. DOI: 10.21873/anticanres.13646

20 Todo M, Shirotake S, Nishimoto K, Yasumizu Y, Kaneko G, Kondo H, Okabe T, Makabe H and Oyama M: Usefulness of implementing comprehensive pharmaceutical care for metastatic renal cell carcinoma outpatients treated with pazopanib. Anticancer Res 39(2): 999-1004, 2019. PMID: 30711987. DOI: 10.21873/anticanres.13205

21 Chang YR, Huang WK, Wang SY, Wu CE, Chen JS and Yeh $\mathrm{CN}$ : A nomogram predicting progression free survival in patients with gastrointestinal stromal tumor receiving sunitinib: Incorporating pre-treatment and post-treatment parameters. Cancers (Basel) 13(11): 2587, 2021. PMID: 34070456. DOI: 10.3390/cancers 13112587

22 Escudier B, Worden F and Kudo M: Sorafenib: key lessons from over 10 years of experience. Expert Rev Anticancer Ther 19(2): 177-189, 2019. PMID: 30575405. DOI: 10.1080/14737 140.2019 .1559058 
23 Piha-Paul SA, Dumbrava EE, Nair BC, Xiong W, Xu L, Mostorino R, Subbiah V, Tannir N, Fu S, Naing A, Janku F, Karp DD, Patel S, Daw NC, Hong D, Meric-Bernstam F and Zinner R: A Phase I trial of the MET/ALK/ROS1 inhibitor crizotinib combined with the VEGF inhibitor pazopanib in patients with advanced solid malignancies. Onco Targets Ther 14: 3037-3049, 2021. PMID: 33994796. DOI: 10.2147/OTT.S291801

24 Ivanyi P, Eggers H, Hornig M, Kasper B, Heissner K, Kopp HG, Kirstein M, Ganser A and Grünwald V: Hepatic toxicity during regorafenib treatment in patients with metastatic gastrointestinal stromal tumors. Mol Clin Oncol 13(6): 72, 2020. PMID: 33005406. DOI: $10.3892 /$ mco.2020.2143

25 Yamaoka T, Kusumoto S, Ando K, Ohba M and Ohmori T: Receptor tyrosine kinase-targeted cancer therapy. Int J Mol Sci 19(11): 3491, 2018. PMID: 30404198. DOI: 10.3390/ijms19113491

26 Brodowicz T, Liegl-Atzwanger B, Penel N, Mir O, Blay JY, Kashofer K, Le Cesne A, Decoupigny E, Wallet J, Hamacher R and Deley ML: Assessing prognostic and predictive biomarkers of regorafenib response in patients with advanced soft tissue sarcoma: REGOSARC study. Cancers (Basel) 12(12): 3746, 2020. PMID: 33322802. DOI: $10.3390 /$ cancers 12123746
27 Heudel P, Cassier P, Derbel O, Dufresne A, Meeus P, Thiesse P, Ranchère-Vince D, Blay JY and Ray-Coquard I: Pazopanib for the treatment of soft-tissue sarcoma. Clin Pharmacol 4: 65-70, 2012. PMID: 23204874. DOI: 10.2147/CPAA.S33195

28 Ettrich TJ and Seufferlein T: Regorafenib. Recent Results Cancer Res 211: 45-56, 2018. PMID: 30069758. DOI: 10.1007/978-3-319-91442-8_3

29 Miyake K, Kiyuna T, Kawaguchi K, Higuchi T, Oshiro H, Zhang Z, Wangsiricharoen S, Razmjooei S, Li Y, Nelson SD, Murakami T, Hiroshima Y, Matsuyama R, Bouvet M, Chawla SP, Singh SR, Endo I and Hoffman RM: Regorafenib regressed a doxorubicinresistant Ewing's sarcoma in a patient-derived orthotopic xenograft (PDOX) nude mouse model. Cancer Chemother Pharmacol 83(5): 809-815, 2019. PMID: 30758647. DOI: 10.1007/s00280-019-03782-w 